\title{
Do-It-Yourself Empowerment as Experienced by Novice Makers with Disabilities
}

\author{
Janis Lena Meissner ${ }^{1}$, John Vines ${ }^{2}$, Janice McLaughlin ${ }^{1}$, \\ Thomas Nappey $^{1}$, Jekaterina Maksimova ${ }^{3}$, Peter Wright ${ }^{1}$ \\ ${ }^{1}$ \{Open Lab | School of Geography, Politics and Sociology\}, Newcastle University, UK, \\ \{j.1.meissner2, p.c.wright, janice.mclaughlin, thomas.nappey\}@ncl.ac.uk \\ ${ }^{2}$ Northumbria University, Newcastle upon Tyne, UK, john.vines@northumbria.ac.uk \\ ${ }^{3}$ University of Dundee, Dundee, Scotland, jekaterinamaksimova92@gmail.com
}

\begin{abstract}
Recent HCI research has highlighted the potential afforded by maker technologies for supporting new forms of DIY Assistive Technology (DIY-AT) for people with disabilities. Furthermore, the popular discourse surrounding both the maker movement and disability is one of democratisation and empowerment. Despite this, critics argue that maker movement membership lacks diversity and that within DIY-AT, it is seldom the people with disabilities who are creating such designs. We conducted a qualitative study that explored how people with disabilities experience the empowering potential of making. We analysed online videos by makers with disabilities and conducted fieldwork at two makerspaces. These informed the design of DIY-Abilities, a series of workshops for people with disabilities in which participants could learn different maker technologies and complete their own maker project. Through analysis of participants' narratives we contribute a new perspective on the specific social and material capacities of accessible maker initiatives.
\end{abstract}

\section{Author Keywords}

Making; DIY; disability; empowerment.

\section{ACM Classification Keywords}

H.5.m. Information interfaces and presentation (e.g., HCI): Miscellaneous.

\section{INTRODUCTION}

A wide range of computer-aided manufacturing tools, prototyping toolkits and smart materials have been introduced to serve an enthusiastic consumer group of nonprofessionals who call themselves makers. For them making is not only about using new technologies, but also about the possibilities of what can be created. The potential ability to design and build any object oneself is the basis for what is

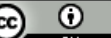

This work is licensed under a Creative Commons Attribution International 4.0 License.

Copyright is held by the owner/author(s).

DIS 2017, June 10-14, 2017, Edinburgh, United Kingdom

ACM 978-1-4503-4922-2/17/06.

http://dx.doi.org/10.1145/3064663.3064674 often referred to as the maker movement. Makers combine hardware and software with digital fabrication and traditional crafts in novel ways [36]. The technological proficiency of non-professionals often comes with a hacker ethos or 'philosophy of sharing, acceptance, and creativity' [34:ix], an ideological underpinning fuelling claims of empowerment [47,53]. Enthusiasts often regard the availability of maker technologies as the first step towards an emancipated prosumer future [57]. However, while personal fabrication can be a reality for those with access to knowledge and tools, not everyone has the same opportunities to take part $[8,54]$. The maker movement has been criticised for lacking demographic diversity [18] and recent reports support the impression that those who selfidentify as makers are quite a homogenous group [61]. Many makerspaces like to envision themselves as welcoming third places in public life [54], established to share tools, knowledge and skills. However, despite best intentions, work is still needed to make these resources accessible to people without a technical background [3].

People with disabilities ${ }^{1}$ are a specific group who could benefit from personal-scale fabrication. Previous research has identified the customisation of commercial assistive technologies and the design of privately manufactured aids as promising approaches to overcome the shortcomings of one-design-meets-all products [31]. Such non-professional initiatives to customise or create assistive technologies have become known under the umbrella term Do It Yourself Assistive Technology (DIY-AT). A growing body of HCI research has investigated the applicability and quality of personal-scale fabrication for assistive technology (e.g. $[14,16,29,31])$. However, hardly any prior work has engaged those with disabilities as makers themselves. This is potentially problematic, contradicting the discourse of democratisation and empowerment surrounding making.

In this paper, we describe a qualitative study which considered the ways that people with disabilities already do and can make use of digital fabrication tools. Our research was performed in two phases: first, an exploratory phase

\footnotetext{
${ }^{1}$ This paper uses person-first language instead of identity-first language. While being aware of the criticisms of an exclusive person-first language use [21], for consistency we refer to "people with disabilities" instead of "disabled people".
} 
which involved analysis of online videos created by makers with disabilities, and participant observation activities at two makerspaces; second, we designed and held DIYAbilities, a workshop series inviting people with disabilities to learn how to use digital fabrication tools and complete their own personal maker project. Across both phases of research, we focussed our attention on narratives of empowerment related to making practices. The findings from our study contribute to the emerging literature on DIY-AT by highlighting the different forms of empowerment that might come from the maker experiences of people with disabilities.

\section{DO IT YOURSELF ASSISTIVE TECHNOLOGY}

Hurst and Tobias first introduced the term Do It Yourself Assistive Technology (DIY-AT) to HCI, noting the longstanding problem of high abandonment rates of off-shelf AT and the potential for DIY technologies to support greater adaptation and adoption [31]. Since then, most research in the context of DIY-AT has continued to be motivated by different shortcomings of mass-produced assistive technology. Beyond referring to overall high abandonment rates $[29,30,42]$, the relative high costs of commercial designs and their lack of adaptability to individual preferences have been criticised $[14,16,45,46]$. Within this work different factors related to the adoption of DIY-AT have been attended to. For example, Hook et al. [29] highlighted a range of challenges related to DIY-AT from the perspective of the care network around children with disabilities. Rajapakse et al. [46] investigated a collaborative approach through the lens of infrastructuring where the main challenge was in bringing together different communities with the necessary skills for producing DIYAT (e.g. makers and disability service organisations). Finally, Buehler et al. [14] examined the skillsets of an existing online community of DIY-AT designers and their motivations to share their inventions on Thingiverse, a popular open-source repository of 3D CAD models.

The above studies emphasise how rapid prototyping tools hold great potential for producing individualised, and affordable, AT. However, despite use of the DIY acronym, people with disabilities have been framed as primarily users or consumers, rather than producers, of DIY-ATs. For example, Hook et al. defined DIY-AT generally as 'the development of AT by non-professionals' [29:598]referring to parents, friends and care-givers. Buehler et al. [14] noted that it is rarely the people with disabilities themselves who create and share DIY-AT designs online. While DIY is not an unfamiliar concept in AT, it seems to be mainly understood as a practice of able-bodied people with a technical background who are part of the extended care network around a person with a disability. There are only few studies that have directly acknowledged the possibility of an active role for people with disabilities. For instance, Bennet et al. [9] and Hofman et al. [28] discussed the implications of involving prosthetics users as codesigners. Hurst and Kane discussed how making can be made more accessible for novices with and without disabilities [30]. Buehler et al. expanded on this by exploring specific obstacles in the context of 3D printing in special education [15]. However, while these approaches do acknowledge more agency of those using DIY-AT, they still maintain a certain dependency on either co-designing together with (able-bodied) makers or on additional modifications of standard maker equipment.

\section{Shifting Views and Disability Models}

Further insights on DIY-AT in relation to empowerment can be found in the disability studies literature. There is a long lineage of work defining, critiquing and developing different models of and attitudes towards disability. It has been claimed that many societies' responses to disability are akin to a Medical Model $[33,40]$, where an individual's physical, sensory or cognitive deficit is construed to be a private 'tragedy' [6] which needs to be 'fixed' [38,39]. With good health defined by the absence of sickness, a medical approach to disability runs the risk of reducing people with disabilities to functional differences compared to "normal" people's physical or cognitive abilities $[22,33,40]$. This leads to presumptions which run the danger of setting a person's disability as synonymous with inability [13] and thereby categorically side-lining them.

The activism of the Disabled People's Movement in the 1970s resulted in the rise of the Social Model which depicts disability as a socially constructed form of oppression leading to the social exclusion of people with impairments $[6,55]$. The conceptual debates around distinguishing between disability and impairment marked a paradigm shift that became a powerful tool for political struggle. In a relatively short time it became the point of departure for achievements not only in national and international politics and legislation, but also in technology design (such as web accessibility) [39]. However, despite its successes as an activist tool, the Social Model has also been criticised. Since the late 1990s a variety of critical perspectives have evolved representing theoretical positions spanning materialist to post-modern camps [7,19,25,55,56,58]. A core argument across this work has been a call for more holistic approaches to understanding disability, since it cannot be fully explained by the presence of social barriers. A more holistic model of disability would involve challenging culturally embedded stigmatising ideologies $[49,58]$ and considering the embodied experience of being a person with disabilities [7,25]. Thus, disability and impairment need to be discussed both on an individual experiential level and a social structural level. This is important because every impairment is different and being labelled as disabled in today's society still holds many forms of disadvantages [58], such as limited opportunities for employment [5] or leisure [17].

A small number of HCI researchers have addressed these critical perspectives and discussed their implications for the design of AT (e.g. Frauenberger [22,23] and Mankoff, 
Hayes and Kasnitz [39]). A core argument is that much AT design echoes the medical model, which itself is deeply embedded in society [13]. Disability is regarded as a disruptive characteristic, which prevents a person from doing the same things as a "normal" person (or a designer or a maker). Frauenberger [22] developed a holistic account of disability, implying that disability exists in a complex multi-layered reality affected by both individual/physical and social/structural factors. This conceptual shift is reflected in the emergence of studies that adopt participatory approaches of designing with and for people with disabilities (e.g. [19,20,32,52,53]). These studies show that it is not enough either to fix individual problems (as per the medical model) or to reduce barriers to sociotechnical infrastructure (as per the social model). Rather designers also need to involve people with disabilities in design processes and promote the articulation of lived experiences in order to understand how well-being and agency are understood by themselves. In the context of our study this means investigating how people with disabilities can and do make use of maker technologies themselves - not only as an autonomously applied form of DIY-life hacking but also for experiencing self-efficacy and regaining control over objects in everyday life. It also means looking at how people with disabilities themselves might understand the empowering potential of maker technologies, and using this to potentially challenge the HCI-rhetoric of empowerment surrounding maker culture, which we discuss below.

\section{DIY-Making and the Rhetoric of Empowerment}

In recent years HCI research has been active in highlighting the empowering potential of maker technologies $[2,27,35,42,43,53]$. However, the examples underpinning these arguments often suggest a relatively narrow notion of empowerment. Roedl et al. [47] conducted a discourse analysis on 191 published papers related to maker culture and identified two main categories of celebratory rhetoric. They referred to these as 'materially empowered subjects' and 'means of social progress' [47:9]. The first category included approaches focussing on individuals able to appropriate artefacts, reuse waste materials and develop long-term care for their material possessions. The second highlights the importance of pleasure in creating objects, sharing resources, and critiquing consumer culture. While these themes have helped to establish making as a relevant topic for HCI, Roedl et al. note that the general talk about the potential for empowerment takes the focus away from finding practical approaches to achieving it. They note that 'the celebratory rhetoric around the maker obscures the concrete mechanisms through which making operates as a critique of traditional computing and its relationship to consumer culture' [47:14].

While empowerment is a commonly agreed-on ideal to strive for and ever more HCI researchers pick this explicitly as their aim for design, it comes with some problematic implications. We discuss these in detail below as they have significantly shaped the approach and scope of this study.
First, despite HCI's self-image as an interventionist design field [20,24,44,51] and its good intentions for design [48], it often struggles to move beyond theoretical empowerment through technology. HCI has long benefitted from using the construct of the disempowered user to legitimise itself as a specialised field within computer science by positioning its researchers as advocates for the human in the system $[10,11,26,48]$. However, real empowerment goes beyond advocacy. Ironically, the discrepancy between ideology and practice is particularly pronounced in HCI's accounts on making and DIY $[3,8,47]$. Seeing the maker as the logical antonym to user [47] challenges the role of the designer and their traditional control over design. So far, this tension has often been encountered as a potential site of conflict rather than seeing it as an opportunity to re-think design. For example, rather than reifying the passivity of the user through closed black-box designs, designers could create technologies open for subsequent modifications (if the user wishes to do so) and thereby provide greater opportunities for empowerment [50].

Second, the multiplicity and inaccuracy of arguments for empowerment through making is problematic: there are many definitions of what empowerment might mean in different contexts, and there is often ambiguity on how exactly authors understand this term. It is relatively seldom that authors are as explicit as Mellis and Buechley who refer to empowerment as 'the ability and confidence [for people] to control the technology in their life' [41:1731]. However, this definition could be different in other situations where empowerment is more a matter of achieving social recognition. Thus, it is important to make explicit what exactly is meant by empowerment in the specific context. Furthermore, it should be questioned if this definition needs to be prescribed top-down or if it should be for those to be empowered to define it for themselves.

Third, empowerment is fundamentally paradoxical because it relies on a given power imbalance where one member is in a position to give power to the other $[1,50]$. There is (and always will be) a certain distinction between designer and user, and it still is the designer who needs to give power to the user. While the dilemma cannot be easily overcome, it is important to address it. As designers and researchers who aim to design for empowerment, it is crucial to reflect on the implications of this to identify pragmatic approaches to effectively equalise these power dynamics.

\section{METHODS AND RESEARCH PROCESS}

Our overview of prior work highlighted two key issues: (1) a lack of opportunities for people with disabilities to participate in the design of DIY-AT; (2) a risk of making unfounded claims of empowerment through making (with empowerment being implicitly defined top-down in the researcher's terms). Therefore, we created a maker initiative specifically for people with disability, in which it was up to the participants to explore the empowering capacities of maker technologies. In our research, we were 
interested in how people with disabilities experience selfdirected uses of maker technologies ${ }^{2}$ when provided with the necessary infrastructure, and beyond that, if and how their personal experiences of empowerment would be expressed. These individual accounts could then be related to the identified HCI rhetoric on making and empowerment.

The research applied a two-phase mixed-method approach. Initial exploratory research was conducted online and in makerspaces (phase 1) to inform the design of a series of maker workshops (phase 2). The main rationale for the preparatory work was to identify aspects constituting barriers and enablers for people with disabilities in the context of making activities. This was important to give the able-bodied researchers the opportunity to develop an understanding of potential challenges in participating in maker activities for individuals with disabilities. In a practical sense, phase 1 was needed to make the workshops in phase 2 as accessible as possible. Furthermore, the insights gathered in phase 1 also sensitised subsequent reflections on narratives and experiences.

The qualitative data gathered in these two research phases comprised: user-generated videos and other online data and observational notes in phase 1 , and; transcribed interviews, debriefing conversations and show-and-tell videos created by participants to document their projects in workshops in phase 2. To analyse this broad and diverse data corpus we chose to conduct thematic analysis following Braun and Clarke [12] at different stages of the project. All along the study, the focus of our analysis was on the participants' experiences, the role of different material and social factors for accessible making (e.g. the design of the equipment or the right amount of assistance) and expressions of empowerment. In phase 2, this facilitated the identification of themes re-occurring across different workshops, which could then be related to issues highlighted in phase 1 to further contextualise and draw out insights.

\section{Phase 1: Preliminary exploratory studies}

The first phase of the project was concerned with exploring existing practices of makers with and without disabilities. This was divided into two activities: first, the analysis of online videos by makers with disabilities, and; second, observations conducted in existing makerspaces.

\section{Phase 1a: Analysis of Online Video Content}

First, a qualitative content analysis was conducted on usergenerated and publicly shared online video content which presented DIY-experiences from the perspective of creators with disabilities. We chose to search for videos over other communicative mediums as, following prior work [4], we assumed such content's presentation would provide a resource of insight and first-hand accounts directly from makers with disabilities themselves. For the data collection,

\footnotetext{
${ }^{2}$ Maker technologies refer to both personal-scale fabrication tools (3D printers, laser cutters) and proto-typing toolkits (microcontrollers) in this paper.
}

mainstream online search engines were used in combination with a selection of keywords related to DIY/making and disability. The search results were then manually checked for accounts on DIY activities directly reported by a person who also stated to have an impairment or disability. Sometimes news reports or documentaries would provide further details to search for specific individuals who matched the search criteria. From an initial 17 candidates, three video collections were selected for further analysis:

- Project Unicorn ${ }^{3}$ : the documentation of a 3D-printed arm prosthesis that shoots glitter designed and iteratively improved by a 10 -year old girl with amelia, a birth defect which results in babies born without limbs (9 videos).

- Zebreda makes it work! ${ }^{4}$ a video series featuring the DIY-mindset of a woman with arthrogryposis multiplex congenita (a congenital disability characterized by joint contractures and weak muscles) and demonstrating a variety of self-designed life-hacks (14 videos).

- Paralyzed Living ${ }^{5}$ : video tutorials and practical tips on wheelchair maintenance and accessible home improvement by a man with paraplegia ( 8 videos).

These examples were chosen as they documented DIY activities related to a disability and showed the creators reporting on the process. They also incorporated an interesting variety of maker identities and DIY techniques. The 31 videos, the biographies of the creators, video description texts, and comments constituted the data corpus on which thematic analysis [12] was conducted.

\section{Phase 1b: Makerspace Observations}

While the online content analysis focused on those who perform DIY-practices, this study concentrated on matters of cultures and environments in which making took place. Looking at material aspects of space and equipment as well as the social dynamics within the facilities helped develop a deeper understanding of makerspaces as specific sites of collective learning and practice. The observations were conducted over the course of two visits at each site and were documented in form of written field notes and photos. The observation included looking at the equipment and the types of projects developed at each makerspace, as well as talking with makerspace members about their activities or making in general. Observations also paid attention to the different forms of social interactions between members.

The two spaces were selected as they both self-identified as makerspaces but had different visions on what this exactly meant. Makerspace (A) specialised on supporting a range of high-tech maker activities, such as electronic kits, CNC manufacturing and 3D printers. Makerspace (B) was more akin to an open workshop offering space for traditional

\footnotetext{
${ }^{3}$ www.youtube.com/playlist?list=PL1Wj1zykqXZRTwW-JDtx4bFRQSJ-pg62d (accessed on 25.08.2016)

${ }^{4}$ www.zebredamakesitwork.com/category/at/ (accessed on 25.08.2016)

${ }^{5}$ http://www.youtube.com/user/blackpearlv6/ (accessed on 25.08.2016)
} 
crafts as well as recent maker technologies. These spaces also differed significantly in the types of projects conducted as well as its audience. While (A) was primarily formed by a group of men working on their respective electronic projects, the makers at (B) were dispersed in different rooms for woodworking, painting, repairing old machines, knitting or $3 \mathrm{D}$ printing.

However, the makerspaces also had some aspects in common. Both were founded and run by a small group of dedicated individuals, and both were open to members and visitors during official opening times. Furthermore, the spatial organisation of the infrastructure for both spaces had grown with its members instead of being formally regulated. The spaces were shaped directly by their members, their maker interests and their specific projects. Completed projects were carefully arranged in a 'shop window' while the rest of the rooms were filled with a mixture of tools, materials, work-in-progresses and storage boxes. In both spaces, there were few official rules; apart from opening times, pricelists for materials and tools, and legal health and safety rules, there were mostly implicit best practices in place which makerspace administrators and regulars were happy to share with less experienced members if asked.

\section{Phase 2: DIY-Abilities Maker Workshops}

The second phase of the research consisted of running DIYAbilities, a series of maker workshops for adults with disabilities. The initiative sought to provide a realistic maker setting in terms of technological resources. Thus, participants were offered opportunities to learn how to use different mainstream maker technologies and work on their own maker project.

\section{Workshop Design}

The workshop series was designed to comprise five sessions per participant. Each workshop lasted three hours including breaks and took place in university facilities that had similar equipment to a maker space and were legally required to be accessible. The featured maker technologies were chosen due to their popularity among mainstream maker communities: 3D printing, laser cutting and programming electronics with microcontrollers. All participants were keen to work with 3D printers, but were also enthusiastic to learn about the other technologies. The workshop series was structured so that each participant would first attend three introduction sessions, teaching them to use the maker technologies based on guided tutorials:

- Introduction to $3 D$ printing: Explaining the basics of additive manufacturing and demonstrating the concept with a Makerbot Replicator 2X. Downloading existing 3D models from Thingiverse. Learning basic 3D modelling with TinkerCAD. Session outcomes: Zipper extender (printed from a downloaded 3D model chosen by the facilitators), tick fork (printed from a downloaded file found by the participants on Thingiverse), dice (printed from a 3D model created by the participants).
- Introduction to Laser Cutting: Explaining the basics of subtractive manufacturing. Discussing suitable materials and demonstrating cutting/engraving with a VLS 6.60 laser cutter. Learning the basics of vector graphics and designing in Adobe Illustrator. Session outcomes: Puzzles (topic chosen by the facilitators to demonstrate different software drawing functionalities; individually designed and fabricated by the participants), key rings (topic suggested by the facilitators, individual designs by the participants), decorative plaque (topic by participant).

- Introduction to Electronics: Explaining the basics of circuitry and different components. Peer programming with an Arduino Uno and circuit prototyping on a breadboard. Session outcome: a button-triggered traffic light system consisting of LED-lights and a sound buzzer (the facilitators first showed how to make a LED blink, the participants then chose which other components they wanted to add and implemented the functionality)

The final two sessions were reserved for the participants applying their knowledge and working on an individual maker project based on their own ideas. It was up to the participants to choose the specific topic for their final project. However, given that the workshops were offered exclusively to people with disabilities and that many of the samples produced in the introduction sessions were aligned with the theme of accessibility hacks, it was not surprising that most projects can be categorised as DIY-AT as well.

Specific care was taken to prepare the environment to be as accessible as possible. This included obvious details such as shifting furniture to make space for wheelchairs, providing large screens and magnifiers for attendees with visual impairments and installing a webcam showing the inside of the laser cutter for participants who cannot stand. The accessibility considerations also affected the tutorials, which were designed to use specific materials that might be useful for certain participants' circumstances (e.g. rubbery and glow-in-the-dark 3D printer filament). The careful preparation aimed to provide a comfortable and flexible environment for all who participated. However, it is also important to state that all maker technologies were introduced by their standard way of operation (without modifying their design for accessibility reasons), and that the content of the tutorials would not have been any different if done for an able-bodied audience.

\section{Participants}

Five participants were recruited by circulating a workshop flyer through several local disability-related organisations, institutions, and sports clubs. People interested in participating could then contact the researcher who would provide more details and answer any questions. While three participants reacted directly on the call, two joined in as interested friends to another participant. To accommodate participants' availability, workshop sessions were held on seven different dates distributed over three weeks. This meant that not all participants attended the same sessions, but it allowed at least three participants to attend all five 


\begin{tabular}{|c|c|c|c|}
\hline \multicolumn{2}{|c|}{$\begin{array}{l}\text { Participant description with } \\
\text { pseudonym and background }\end{array}$} & \multirow{2}{*}{$\begin{array}{c}\text { Impairment } \\
\text { motion } \\
\text { speech }\end{array}$} & \multirow{2}{*}{$\begin{array}{c}\text { Attended } \\
\text { Sessions }\end{array}$} \\
\hline Peter & $\begin{array}{l}\text { Full-time student, wheelchair } \\
\text { user with cerebral palsy }\end{array}$ & & \\
\hline James & $\begin{array}{l}\text { Part-time IT employee with } \\
\text { exterior nerve disorder }\end{array}$ & $\begin{array}{l}\text { motion } \\
\text { speech }\end{array}$ & 5 \\
\hline Mary & $\begin{array}{l}\text { Part-time local government } \\
\text { employee with partial vision } \\
\text { and atypical autism }\end{array}$ & $\begin{array}{l}\text { vision } \\
\text { cognition }\end{array}$ & 1 \\
\hline David & $\begin{array}{l}\text { Seeking employment, } \\
\text { cerebral palsy }\end{array}$ & $\begin{array}{l}\text { vision } \\
\text { motion }\end{array}$ & 5 \\
\hline Harry & $\begin{array}{l}\text { Seeking employment, } \\
\text { wheelchair user with cerebral } \\
\text { palsy and dyslexia }\end{array}$ & $\begin{array}{l}\text { motion } \\
\text { speech } \\
\text { cognition }\end{array}$ & 3 \\
\hline
\end{tabular}

Table 1. Participant details.

sessions and complete their projects. As for the other two participants, one decided to only attend one session (as she was busy the other dates), and the other participant was limited by the availability of his carer (and so was unable to take part in the final project sessions). The five participants are listed with their pseudonyms in Table 1. Four participants were male and one female, with their ages ranging from 20 to 47 years. They had a variety of different conditions including cerebral palsy, congenital nerve disorder and atypical autism entailing different levels of visual, motor and speech impairments. Two were wheelchair users and needed personal assistance.

Preparation interviews prior to the workshops served as opportunities to discuss the participants' motivations for taking part, their prior experiences of technology and making, and any specific needs they had regarding the workshop environment. While none of the participants had previous coding or designing skills, all were experienced computer users with a general interest in technology. None of the participants stated to have previously engaged in any form of making. Indeed, apart from a vague idea based on the workshop title and description, none of them were sure what the term making meant. They all had heard of 3D printers, but none had seen one in real life. Most could not remember ever having created anything to help them in their everyday life. However, despite this lack of prior experience, the DIY-Abilities workshops were overall well received by the participants. One participant exclaimed: "I can sum up the workshop in a few words: Awesome, inspiring us to use what ability we have to make something good and great." (David, workshop 7). All the participants showed great interest in the technologies, engaged in a steep learning curve, and were able to fabricate several designs.

\section{Facilitation and Research Process}

The workshops were run by the lead researcher alongside two workshop assistants, who were experienced in digital fabrication and could support the participants with manufacturing and assembling. They also helped with documenting the sessions and creating a comfortable atmosphere. None of the participants had visited a
University research lab before, so they were curious about the different kinds of researchers they were able to meet. Casual chats between participants and workshop assistants were therefore a good way for breaking the ice and settling in while getting to know the new environment.

In addition to the preparatory interviews, data collection was integrated into the workshop process, with the participants consenting to being audio recorded and occasionally being photographed or filmed during the sessions. Show-and-tell-videos were recorded at the end of each session, along with debriefings with the workshop assistants. The former documented the personal narratives of individual maker experiences and how these developed over the five workshops; the latter captured observations from the perspective of different research team members.

\section{PHASE 1 INFORMING PHASE 2}

The studies of phase 1 highlighted several accessibilityrelated issues relevant for preparing the phase 2 workshops.

\section{Expressing maker experiences in videos}

Most of the videos followed a similar format, usually being a demo, show-and-tell or how-to tutorial. They showed the person with disabilities with their project directly in the environment where they would use it. Notably, rather than using a tripod, the filming was usually done by another person who often even played an active role in the video by asking questions. Sometimes this creates an impression that it was rather the film-maker's idea to start the video channel and share the DIY-experiences. Only in one case did the protagonist explicitly state that it was a personal mission to share personal knowledge with people in similar situations. However, all protagonists seemed comfortable with being filmed and to enjoy the recognition for their DIY-activities.

Based on these insights, we decided to use show-and-tell videos as part of the data collection during phase 2 . The production of these videos was highly influenced by the analysed formats; it was the researcher filming the participant from a perspective previously agreed with them (this was mostly close-ups on the created artefacts) and giving prompts in form of open questions.

\section{Accessibility issues in makerspaces}

Initially, the makerspace observations served as a general accessibility check of these sites. Both makerspaces had a seemingly narrow definition of accessibility, which only considered the building. Makerspace (A) had a ramp to its entrance while (B) had a stair lift inside. However, it was clear that these accessibility-features were likely not used frequently by people with disabilities: heavy entrance doors were hard to open (A) and the stair lift was blocked with piles of material (B). Perhaps unsurprisingly considering these issues, no person with a visible or disclosed disability was met during the fieldwork. These observations were consistent with the impressions from the online videos, where the DIY practices were seen to be performed in people's homes rather than in community facilities. 
Apart from issues with the accessibility of the material infrastructure, the observations also pointed out the importance of accessibility on a social level. Both spaces emphasised their welcoming culture towards anyone interested in making. However, with all the complicated looking technologies and the confident regulars around, makerspaces can feel intimidating for newcomers. It was thus decided to conduct the maker workshops not in a makerspace but instead at university facilities where it would be easier to create a sensitive atmosphere without the established social dynamics in a makerspace.

\section{Accessibility as a motivation for making}

Issues of accessibility were seldom explicitly mentioned in the videos, but were always implicitly present. As an important part of the life experience of the protagonists, their DIY-projects often addressed issues within their local environment that posed barriers for them. Rather than problematising their own disability, the protagonists were very clear about how their projects were dealing with external matters. For example, when Zebreda explained her reasons for inventing the "Door Hook Opener" she first described the problem by referring to the door and wheelchair before mentioning her limited motion range: "Because when you enter the room, the handle was on the right hand side and that was too deep into the corner. Because of my wheelchair and its front being too long, I couldn't get close enough. And my arms don't reach out far enough to reach the door knob in the corner. ",

This specific insight sensitised later reflections on the project outcomes in phase 2 . Above we noted that it was not surprising that most participants chose a project related to DIY-AT due to the way the workshop series was structured. However, another way to look at it could be to regard this as an expression of intrinsic motivation to hack the able-ist design of the everyday environment which is (sometimes subconsciously) based on the designer's experience.

\section{FINDINGS: EXPERIENCES OF MAKING}

Making is generally a complex and time-consuming process consisting of many different steps and activities, which pose challenges to any novice regardless of individual abilities. Having limited dexterity when building circuits of tiny components, being partial-sighted when using high-end CAD software with small icons or needing more time to memorise different sequences of fabrication steps obviously adds to the complexity. A workshop assistant recounted how three participants were struggling with a 3D modelling tutorial: "David couldn't see and read the instructions. That's why when Peter asked him for assistance with the key chain he was like 'No, because I don't see.' So he cannot see the exercise properly. He can't manage to do it by himself. [...] Harry, he also couldn't do much work just because he couldn't move his hands." (workshop 2). Using excerpts from across the whole data corpus, we identified four main themes around experiences

\footnotetext{
${ }^{6}$ https://youtu.be/hYxKknar5rY, 0:46-1:04, (accessed on 15.08.2016)
}

of making with disabilities and strategies participants used to overcome challenges with learning and using the technologies in the workshops.

\section{Pragmatism and Patience}

The participants were very pragmatic in how they coped with the general difficulty of the tasks and specific challenges related to their impairment. For example, there were several issues with the interaction modalities of the technologies not suiting the participants' abilities. With limited dexterity, certain keyboard shortcuts requiring two hands could not be used, or placing tiny electronic components into a breadboard was difficult. While these challenges slowed the process down, these were no reasons to give up. Patience seemed to be a key virtue and was mentioned in several show-and-tell videos. For example, Peter who had just learned to use a mainstream 2D CAD software to design for laser cutting stated that it had been "[n]ot that difficult if you took a bit of time" (workshop 1). A similar approach was mentioned in workshop 3 by James and Harry who had teamed up for learning how to program a microcontroller: "It took some persevering to get there, because the buzzer wouldn't work at first, and then we had to change the code five or six times." (Harry); "A lot of debugging was done." (James)

\section{Collaboration for Independence}

Depending on the number of participants in each workshop, they would work individually or in teams of two or three (including a workshop assistant) on a computer. This worked well, although the teamwork also came with tradeoffs. Two participants sitting at one computer implied that one of them got to do more than the other, and that the work on occasion could become less focussed. However, it was found that the participants used collaboration among themselves as a means to create productive coalitions to increase their independence from the able-bodied workshop facilitators. They were considerate of each other's strengths and weaknesses and actively negotiated who was responsible for different tasks. For example, James and Harry teamed up for the introduction session on laser cutting (workshop 4) and distributed tasks according to their abilities. While James can only use his right-hand side, Harry has dyslexia and over-all limited motor skills. Harry therefore took control over the mouse (his preferred input device), and James operated the keyboard and made sure that the right succession of steps were completed. James would do this in a very considerate way, slowing down the pace and choosing to work on Harry's project first. This involved James inviting Harry into the making and learning process by leaving aesthetical decisions to him; James would then guide him verbally and give hints on how to use his mouse for drawing what he had articulated as wishing to do. This facilitated better learning on Harry's side, while it slowed down James's progress on his own keyring design.

Over time, the participants became increasingly confident in negotiating their own role and were more comfortable with the technology as the challenges were shared with a 
peer. These positive social effects came through particularly strongly in the show-and-tell videos of Peter, a participant with a quite severe speech impairment. While his first and individual videos mainly consisted of briefly answering with "yes" and "no", he elaborated in more detail on the making process in the video he did together with David. After that session (workshop 3) he was more outgoing, even in the documentation of his individual project where he would often elaborate on his affirmative answers: "I made a tray to hold my Boccia balls. [...] I designed it on Adobe Illustrator and used a laser cutter to cut it into wood." (Peter, workshop 5) - "Was it a lot of work?" (lead researcher) - "Yes, designing the interlocking.” (Peter)

\section{Developing New Abilities}

In the preparation interviews the participants were asked what they were hoping to get out of the workshops. While not stating it explicitly as an expectation, three participants mentioned that learning new skills was particularly significant in their difficult hunt for paid work. James stated for example that he was hoping for "[s]kills in areas that you'd be doing basically. Just like have basic skills in these areas and if they could be used in my future job hunting skills then so be it. But, I'm [open to] learning new stuff and improving myself basically" (James, pre-interview). As the workshops progressed, making was increasingly perceived as a potential extension of their own abilities. In the preparation interview Mary said she was not good at drawing and reported her previous negative experiences with graphic design programs. She was impressed with how easily she could produce five beautiful artefacts with a professional vector graphics editor program and a laser cutter in just three hours. In the show-and-tell video she emphasised how the software would help her creating her own design: "I used a program which helped me to create shapes, to create text which could be engraved, and [I] also imported a picture from the internet which was engraved as well." (workshop 5).

\section{Material Points of Contact}

The created artefacts were of social significance as well. As could be observed in the makerspaces, the work in progress often helped members to approach others who they did not know so well and start conversations. For example, the lead researcher wrote the following in her observation notes at makerspace (B): "A man was sitting in the electronics corner working on a small but heavy looking machine. He explained that this was a vacuum pump that he tried to get running with the electricity network of the space. Another man joined us and asked, pointing at the machine, if we had a 'repair cafe' event going on. The man with the vacuum pump answered: 'Here at the space, we have a reparation café every day! "' In this way, the objects can serve as material points of contact that lower the barrier for social interactions. In the workshops a similar situation was observed by an assistant in relation to the tools used in his teamwork with Peter: "I was sat here and [...] we were just passing [the components] round, so he wasn't saying that much, but then when I sat in the middle and he took over the [...] mouse, he had a clear role then and then a few things, like, oh can you scroll up? Can you scroll down? And because he's the only one with the mouse so he has to do it and then it was kind of through that that you kind of start a conversation." (workshop 3). With Peter controlling the mouse and the assistant occasionally helping with typing, their social interaction was initialised and channelled through coordinating the making process, gradually evolving into other chats not related to their work. This special status of the objects and tools is of course not exclusive to the context of makers with disabilities, but such conversation starters could be of particular significance when collaboration is required and people are not used to interacting with peers with impairments.

\section{DISCUSSION: OBJECTS FOR EMPOWERMENT}

Besides verbal statements narratives can also be embedded in artefacts [32,52]. The show-and-tell-videos showed that there is more to a self-made object than just its materiality. The artefact descriptions often referred to its provenance and personal significance. Talking about the self-made artefact revealed a multiplicity of associations. For instance, in their show-and-tell video for the electronics introduction session Peter and David would not only present their interactive traffic-light system but also refer to its code, its components, its functionality and its flaws: "We made a traffic light. I'll show you, and coded it on a computer. [...] It goes green and red with a delay in between, and then a buzzer." (Peter) - 'Yes, and we've got a button. So we've got the equipment set up, we've cabled it, and using the system from the computer we've programmed it to say what we want it to do, the time we want it to do it, and in which sequence we'd like it to do it. And having it to be able to stop without going on for too long." (David, workshop 3) In this way, one and the same object could represent an idea, a creation process, aesthetic choices, learning achievements, references to other parts of their identity, and a material reward for their efforts.

Similarly, the creations by the three participants in the final project sessions demonstrated a variety of ideas and different approaches to the making process. The individual projects (cf. Figure 1) comprised: the boccia ball holder by Peter, a storage extension for the armrest of a manual sports wheelchair; the temperature sensor by James, a safety device to detect heat for other people with disabilities; and the award by David, a modular trophy object on a base with integrated lights. The projects demonstrated how the participants explored the mentioned theme of ability extension in various forms of individual empowerment. The diversity of projects represented different personal priorities within that space. Based on such materialised narratives we will now revisit how the artefacts represent three different notions of empowerment, how each of these relate to the rhetorical strategies identified by Roedl et al. [47] and how these particular instances highlight different perspectives on a theoretical concept of empowerment.

\section{The Boccia Ball Holder (Taking Personal Action)}

Peter used his new maker skills to 'hack' a solution to an accessibility issue in his hobby of playing boccia. He uses an electrical wheelchair in most parts of his daily life, which he navigates with a joystick on his left-hand side. Since his left is also his throwing arm, the steering interface 


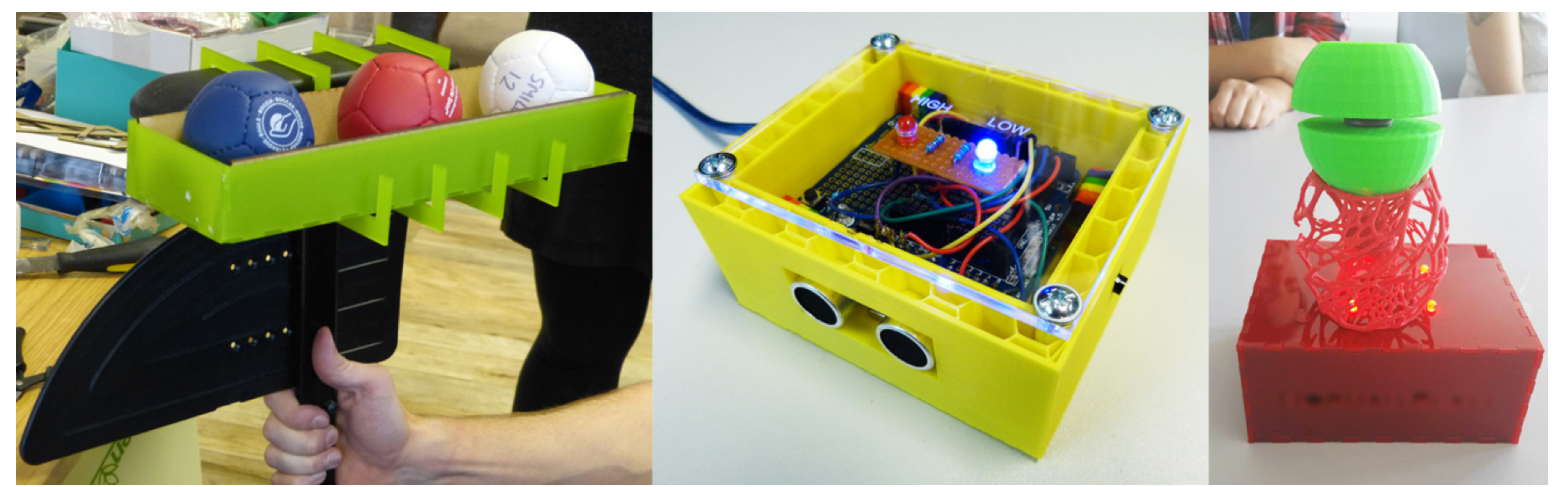

Figure 1. The final maker projects, from left to right: the boccia ball holder, the temperature sensor and the award.

blocks his way and he needs to use a manual wheelchair for his sports. However, this causes him to require assistance for getting the balls for each play. Since he does not like to rely on other people, Peter created the boccia ball holder, a storage extension for the armrest of his sports wheelchair. He designed the object to hold four boccia balls, which should relieve him from needing to be moved to the ball stand each play and significantly increase Peter's personal autonomy in his sports. In this way, his maker project was a practical way of action for self-empowerment. He created an assistive object for himself which does not seem to have an equivalent yet in the existing offer of wheelchair sports equipment. This relates to the identified rhetoric in Roedl et al.'s analysis, which refers to 'makers actively resist[ing] or critique[ing] consumer culture' [47:12]. However, in this case the chosen hack was a result of pragmatism rather than dogmatic reasons. Peter never explicitly criticised the market for not serving his needs, but the his project implicitly did so by signalling that a boccia ball holder was something that he needed and desired. Making was an act of taking control over disempowering situations by using the skills learned. The themes of overcoming challenges with pragmatism and patience and using collaboration to achieve goals gain particular relevance in this context.

\section{The Temperature Sensor (Enabling Others)}

James invented the temperature sensor, an interactive safety device measuring and indicating dangerous heat. His maker project had a similar practical approach to Peter's, with an emphasis on functionality. But in contrast to Peter's project, James developed a design idea not for himself but for other people with disabilities. In this way, he made use of his new maker skills for repositioning his own social role from the one to be helped to the one who helps. The temperature sensor was designed as a portable gadget which should not only measure the temperature, but also give a simple representation of the readings for "a disabled person who would need something like this" (James, workshop 7). James implemented two alternative interaction modalities using a button and a motion sensor that should make it useable for people with different abilities. This approach resonates to a certain extent with the HCI rhetoric of makers 'modify[ing] products to suit their purposes' [47:9] - just that the object to modify is the product the maker had developed himself and that the purposes are defined by an assumed user group in this case. This rhetorical category also highlights how makers see objects as 'unfinished', an aspect which was particularly pronounced in James's project. He reported that he was inspired by the challenge to work with the more sophisticated electronics components. Relating to the theme of developing new abilities, James actively sought new challenges for not only improving his artefact but also his own maker skills. He even had ideas for future design iterations that would transform the device from its current shape as a $3 \mathrm{D}$-printed box into a wearable technology using thermochromatic fabrics. James intentionally placed his focus on the making process and his personal progress as a maker, and aligned this with an agenda to help others and thereby empower himself.

\section{The Award (Demonstrating Skill)}

David created the award, a decorative design consisting of modular re-configurable shapes. In contrast to Peter and James's creations, his project had its main purpose in aesthetic exploration and the showcase of skill rather than being of any functional use. He was eager to integrate all three maker techniques, so that he could show others what he had learned in the workshops. As soon as the award was finished he took photos of it and posted them on his private social media channels. In this way, he proactively created an object that was intended to serve as a material point of contact. By materialising his own maker expertise through his project, David directly aimed at gaining recognition for his new skills. This move mostly relates to the HCI rhetoric that 'acts of making can enhance an object's personal meaning' [47:10]. The technical challenges faced, the invested effort to make it work and personal learning achievements all contributed to developing such personal meanings. By sharing these with others he turned it into an explicit narrative of empowerment.

\section{EXPERIENCE-CENTRED EMPOWERMENT}

The three different approaches to making illustrated in the participants' projects highlight how both the final object and the process of developing it motivated the makers and led to different subjective experiences of empowerment. Challenges within the making process were perceived as opportunities to add meaning to their maker projects. Apart from the differences in ideas and purposes, the objects also 
differed in the materials and technologies used. The boccia ball holder was fabricated using only 2D CAD design and the laser cutter, while the other projects integrated all three techniques. However, all of the show-and-tell accounts expressed 'pleasure of making' [47:11], another rhetoric that Roedl et al. had identified as a general human characteristic with socially progressive implications. The argument here is that since '[c]reating something with one's own hands [..] is often described as a universally pleasurable experience' [47:11], it has the potential to 'gather widespread participation, and to even develop into a mainstream cultural practice' [47:11].

We can compare these insights to Agre's general definition of empowerment as 'a normative form of human organisation [that] is held to both presuppose and encourage a perfect clarity of individual consciousness through participation in large-scale collective action' [1:171]. In other words, empowerment involves three important components: i) an empowering party needs to proactively create an occasion through which another can participate and be empowered; ii) the empowering party needs to provide access to the necessary means of empowerment both materially and intellectually; and iii) the party to be empowered needs to realise that they possess the capacity to make use of the provided resources and embody this as a new part of their identity. We can apply this concept by viewing the DIY-Abilities workshops as a 'normative form of human organisation' which (i) provided access to maker technologies and (ii) motivated the participants by teaching and assisting them when needed. However, while these mainly constituted study design decisions, empowerment only took place (iii) in the specific ways how the participants understood, internalised and expressed their new maker capacities: Peter placed emphasis within his participation to act on a problem; James used his awareness of his new skills to help others; and David gained social recognition through showcasing his maker achievements. The DIY-Abilities workshops enabled the participants to take on a new maker identity that allowed them to act on individual accessibility issues, use their skills to help others and show their expertise.

Reflecting on our findings we can further complicate the issues around designing for empowerment we raised in our discussion of related work. While Peter oscillated between user and designer, James did not plan to use his own creation and thus still operated within a binary. This adds complexity to rethinking the roles and power imbalance within design. The very concept of empowerment implies a separation of the empowered from the disempowered. Yet, this boundary became increasingly blurred during the close collaboration in the workshops where helping each other did not always fall along lines of ability. While we cannot know if working collaboratively was the participants' preferred mode of making, the DIY-Abilities workshops and their outcomes may help to enrich the HCI perspective on what it means to achieve empowerment.

\section{LIMITATIONS OF STUDY AND FUTURE WORK}

In this study, we set out to explore the ways in which people with disabilities might be more directly engaged as active agents in making and DIY-AT processes. While our study has highlighted challenges associated with the material and social accessibility of certain makerspace environments, our DIY-Abilities workshops made space for learning new skills, collaborating among themselves and expressing empowerment in their own terms. However, the study comes with its limitations. Foremost of these is the small number of participants involved in the workshops, all of whom responded to an advert and thus likely already motivated to learn and engage. Furthermore, while dealing with accessibility issues was important for this work, our intention was not to define specific guidelines for how such maker workshops should be run. We note that there will always be contextually specific conditions that impact such initiatives. While our participants could work well with inexpensive workarounds, other participants with different abilities will experience different challenges. Workshop organisers will always need to find their own ways to adapt the available equipment to the participants' needs. However, by reporting our specific approach to the design and set-up of the workshop series, we hope to provide an initial reference point for others who wish to run accessible maker initiatives. Future work could refine this to a set of practical recommendations adding to those on $3 \mathrm{D}$ printing in special education by Buehler et al. [15].

\section{CONCLUSIONS}

This paper has described a study that directly learned from the experiences of people with disabilities engaging with maker technologies. While facing various challenges, the participants were able to use different mainstream maker technologies for their own projects. Several specific potentials for empowerment were identified in the reported experiences. The participants expressed how they saw relevance in learning maker skills for extending their own abilities for self-directed accessibility hacks, utilising their skills for helping others or gaining recognition by showcasing their maker expertise. Indeed, the study results support some of the claims of empowerment through making. However, it is important to consider that empowerment was more a matter of the participants' individual agendas than of the technologies themselves.

\section{ACKNOWLEDGEMENTS}

The authors thank the participants and workshop assistants. This research was funded through the EPSRC CDT in Digital Civics (EP/L016176/1). Data supporting this publication is openly available under an 'Open Data Commons Open Database License'. Additional metadata are available at: http://dx.doi.org/10.17634/154300-43. Please contact Newcastle Research Data Service at rdm@ncl.ac.uk for access instructions.

\section{REFERENCES}

1. Philip E. Agre. 1994. From high tech to human tech: Empowerment, measurement, and social studies of 
computing. Computer Supported Cooperative Work (CSCW) 3, 2: 167-195. https://doi.org/10.1007/BF00773446

2. Binaebi Akah and Shaowen Bardzell. 2010. Empowering products. In Proceedings of the 28th of the international conference extended abstracts on Human factors in computing systems - CHI EA '10, 4021. https://doi.org/10.1145/1753846.1754096

3. Morgan G. Ames, Jeffrey Bardzell, Shaowen Bardzell, Silvia Lindtner, David A. Mellis, and Daniela K. Rosner. 2014. Making cultures. In Proceedings of the extended abstracts of the 32nd annual ACM conference on Human factors in computing systems - CHI EA '14, 1087-1092. https://doi.org/10.1145/2559206.2579405

4. Lisa Anthony, Yoojin Kim, and Leah Findlater. 2013. Analyzing user-generated youtube videos to understand touchscreen use by people with motor impairments. In Proceedings of the SIGCHI Conference on Human Factors in Computing Systems - CHI '13, 1223. https://doi.org/10.1145/2470654.2466158

5. Colin Barnes. 2000. A working social model? Disability, work and disability politics in the $21 \mathrm{st}$ century. Critical Social Policy 20, 4: 441-457. https://doi.org/10.1177/026101830002000402

6. Colin Barnes. 2012. Understanding the Social Model of Disability. In Routledge Handbook of Disability Studies, Nick Watson, Alan Roulstone and Carol Thomas (eds.). Routledge, 12-29.

7. Ana Bê. 2012. Feminism and Disability: A Cartography of Multiplicity. In Routledge Handbook of Disability Studies, Nick Watson, Alan Roulstone and Carol Thomas (eds.). Routledge, London, 363-375.

8. Jonathan Bean and Daniela Rosner. 2014. Making: Moevement or Brand? interactions 21, 1: 26-27. https://doi.org/10.1145/2541669

9. Cynthia L. Bennett, Keting Cen, Katherine M. Steele, and Daniela K. Rosner. 2016. An Intimate Laboratory? In Proceedings of the 2016 CHI Conference on Human Factors in Computing Systems - CHI '16, 1745-1756. https://doi.org/10.1145/2858036.2858564

10. Susanne Bødker. 2006. When second wave HCI meets third wave challenges. In Proceedings of the 4th Nordic conference on Human-computer interaction changing roles - NordiCHI '06, 1-8. https://doi.org/10.1145/1182475.1182476

11. Claus Bossen, Christian Dindler, and Ole Sejer Iversen. 2010. User gains and PD aims. In Proceedings of the 11th Biennial Participatory Design Conference on PDC '10, 141. https://doi.org/10.1145/1900441.1900461

12. Virginia Braun and Victoria Clarke. 2006. Using thematic analysis in psychology. Qualitative Research in Psychology 3, 2: 77-101.

https://doi.org/10.1191/1478088706qp063oa

13. Simon Brisenden. 1986. Independent Living and the Medical Model of Disability. Disability, Handicap \& Society 1, 2: 173-178. https://doi.org/10.1080/02674648666780171

14. Erin Buehler, Stacy Branham, Abdullah Ali, Jeremy J. Chang, Megan Kelly Hofmann, Amy Hurst, and Shaun K. Kane. 2015. Sharing is Caring. In Proceedings of the 33rd Annual ACM Conference on Human Factors in Computing Systems - CHI '15, 525-534. https://doi.org/10.1145/2702123.2702525

15. Erin Buehler, Niara Comrie, Megan Hofmann, Samantha McDonald, and Amy Hurst. 2016. Investigating the Implications of 3D Printing in Special Education. ACM Transactions on Accessible Computing 8, 3: 1-28. https://doi.org/10.1145/2870640

16. Erin Buehler, Amy Hurst, and Megan Hofmann. 2014. Coming to grips. In Proceedings of the 16th international ACM SIGACCESS conference on Computers \& accessibility - ASSETS '14, 291-292. https://doi.org/10.1145/2661334.2661345

17. Liz Carr. 2004. Leisure and Disabled People. In Disabling Barriers - Enabling Environments, John Swain, Sally French, Colin Barnes and Carol Thomas (eds.). SAGE Publications.

18. David Cuartielles, Jonathan Bean, and Daniela Rosner. 2015. Conversations on making. interactions 22, 1: 22 24. https://doi.org/10.1145/2685366

19. Berth Danermark and Lotta Coniavitis Gellerstedt. 2004. Social justice: redistribution and recognition-a non-reductionist perspective on disability. Disability \& Society 19, 4: 339-353. https://doi.org/10.1080/09687590410001689458

20. Lynn Dombrowski, Ellie Harmon, and Sarah Fox. 2016. Social Justice-Oriented Interaction Design. In Proceedings of the 2016 ACM Conference on Designing Interactive Systems - DIS ’16, 656-671. https://doi.org/10.1145/2901790.2901861

21. Dana S Dunn and Erin E Andrews. 2015. Person-first and identity-first language: Developing psychologists' cultural competence using disability language. American Psychologist 70, 3: 255-264. https://doi.org/10.1037/a0038636

22. Christopher Frauenberger. 2015. Disability and Technology: A Critical Realist Perspective. In ASSETS '15 Proceedings of the 17th International ACM SIGACCESS Conference on Computers \& Accessibility, 89-96. https://doi.org/10.1145/2700648.2809851

23. Christopher Frauenberger. 2015. Rethinking autism and technology. interactions 22, 2: 57-59. https://doi.org/10.1145/2728604 
24. Christopher Frauenberger, Judith Good, Geraldine Fitzpatrick, and Ole Sejer Iversen. 2015. In pursuit of rigour and accountability in participatory design. International Journal of Human-Computer Studies 74: 93-106. https://doi.org/10.1016/j.ijhcs.2014.09.004

25. Rosemarie Garland-Thomson. 2011. Misfits: A Feminist Materialist Disability Concept. Hypatia 26, 3: 591-609. https://doi.org/10.1111/j.15272001.2011.01206.x

26. Judith Gregory. 2003. Scandinavian Approaches to Participatory Design. International Journal of Engaging Education 19, 1: 62-74. Retrieved from http://www.ijee.dit.ie/contents/c190103.html

27. Shannon Grimme, Jeffrey Bardzell, and Shaowen Bardzell. 2014. "We've conquered dark." In Proceedings of the 8th Nordic Conference on HumanComputer Interaction Fun, Fast, Foundational NordiCHI' 14, 431-440. https://doi.org/10.1145/2639189.2641204

28. Megan Hofmann, Jeffrey Harris, Scott E. Hudson, and Jennifer Mankoff. 2016. Helping Hands. In Proceedings of the 2016 CHI Conference on Human Factors in Computing Systems - CHI'16, 1769-1780. https://doi.org/10.1145/2858036.2858340

29. Jonathan Hook, Sanne Verbaan, Abigail Durrant, Patrick Olivier, and Peter Wright. 2014. A study of the challenges related to DIY assistive technology in the context of children with disabilities. In Proceedings of the 2014 conference on Designing interactive systems DIS '14, 597-606. https://doi.org/10.1145/2598510.2598530

30. Amy Hurst and Shaun Kane. 2013. Making "making" accessible. Proceedings of the 12th International Conference on Interaction Design and Children - IDC '13: 635. https://doi.org/10.1145/2485760.2485883

31. Amy Hurst and Jasmine Tobias. 2011. Empowering individuals with do-it-yourself assistive technology. In Proceedings of the 13th international ACM SIGACCESS conference on Computers and accessibility ASSETS 11, 11-18. https://doi.org/10.1145/2049536.2049541

32. Tim Ingold. 2013. Making: Anthropology, Archaeology, Art and Architecture. Routledge, London.

33. Marie Johnston. 1996. Models of disability. Physiotherapy Theory and Practice 12, 3: 131-141. https://doi.org/10.3109/09593989609036429

34. Adam Kemp. 2013. The Makerspace Workbench: Tools, Technologies, and Techniques for Making. Maker Media, Incorporated.

35. Stacey Kuznetsov and Eric Paulos. 2010. Rise of the expert amateur. In Proceedings of the 6th Nordic Conference on Human-Computer Interaction
Extending Boundaries - NordiCHI '10, 295. https://doi.org/10.1145/1868914.1868950

36. David Lang. 2013. Zero to Maker: Learn (Just Enough) to Make (Just About) Anything. Maker Media, Incorporated.

37. Stephen Lindsay, Katie Brittain, Daniel Jackson, Cassim Ladha, Karim Ladha, and Patrick Olivier. 2012. Empathy, participatory design and people with dementia. In Proceedings of the 2012 ACM annual conference on Human Factors in Computing Systems CHI'12, 521. https://doi.org/10.1145/2207676.2207749

38. A. Llewellyn and K. Hogan. 2000. The Use and Abuse of Models of Disability. Disability \& Society 15, 1: 157-165. https://doi.org/10.1080/09687590025829

39. Jennifer Mankoff, Gillian R. Hayes, and Devva Kasnitz. 2010. Disability studies as a source of critical inquiry for the field of assistive technology. In Proceedings of the 12th international ACM SIGACCESS conference on Computers and accessibility - ASSETS '10, 3. https://doi.org/10.1145/1878803.1878807

40. Deborah Marks. 1997. Models of disability. Disability and Rehabilitation 19, 3: 85-91. https://doi.org/10.3109/09638289709166831

41. David A Mellis and Leah Buechley. 2014. Do-ityourself Cellphones: An Investigation into the Possibilities and Limits of High-tech Diy. Proceedings of the SIGCHI Conference on Human Factors in Computing Systems: 1723-1732. https://doi.org/10.1145/2556288.2557309

42. Argyro Moraiti. 2015. Empowering Occupational Therapists with a DIY-toolkit for Smart Soft Objects. Proceedings of the 9th International Conference on Tangible, Embedded, and Embodied Interaction - TEI '15: 387-394. https://doi.org/10.1145/2677199.2680598

43. Catarina Mota. 2011. The rise of personal fabrication. In Proceedings of the 8th ACM conference on Creativity and cognition - C\&C '11, 279. https://doi.org/10.1145/2069618.2069665

44. Hafeni Mthoko and Caroline Khene. 2015. Assessing outcome and impact. In Proceedings of the Seventh International Conference on Information and Communication Technologies and Development ICTD '15, 1-4. https://doi.org/10.1145/2737856.2737884

45. Ravihansa Rajapakse, Margot Brereton, Paul Roe, and Laurianne Sitbon. 2014. Designing with People with Disabilities : Adapting Best Practices of DIY and Organizational Approaches. 519-522. https://doi.org/10.1145/2686612.2686694 
46. Ravihansa Rajapakse, Margot Brereton, Laurianne Sitbon, and Paul Roe. 2015. A Collaborative Approach to Design Individualized Technologies with People with a Disability. In Proceedings of the Annual Meeting of the Australian Special Interest Group for Computer Human Interaction on - OzCHI '15, 29-33. https://doi.org/10.1145/2838739.2838824

47. David Roedl, Shaowen Bardzell, and Jeffrey Bardzell. 2015. Sustainable Making? Balancing Optimism and Criticism in HCI Discourse. ACM Transactions on Computer-Human Interaction 22, 3: 1-27. https://doi.org/10.1145/2699742

48. Yvonne Rogers and Gary Marsden. 2013. Does he take sugar? interactions 20, 4: 48. https://doi.org/10.1145/2486227.2486238

49. Margrit Shildrick. 2012. Critical Disability Studies. In Routledge Handbook of Disability Studies, Nick Watson, Alan Roulstone and Carol Thomas (eds.). Routledge, 30-41. https://doi.org/10.4324/9780203144114.ch3

50. Cristiano Storni. 2014. The problem of de-sign as conjuring. In Proceedings of the 13th Participatory Design Conference on Research Papers - PDC '14, 161-170. https://doi.org/10.1145/2661435.2661436

51. Cristiano Storni. 2015. A personal perspective on research through design. interactions 22, 4: 74-76. https://doi.org/10.1145/2786974

52. Megan Strickfaden and Paul A. Rodgers. 2004. "Scripting" - Personal narratives in the designing of artefacts. The Design Journal 7, 1: 3-15. https://doi.org/10.2752/146069204789355227

53. Joshua G. Tanenbaum, Amanda M. Williams, Audrey Desjardins, and Karen Tanenbaum. 2013. Democratizing technology. In Proceedings of the SIGCHI Conference on Human Factors in Computing Systems - CHI '13, 2603. https://doi.org/10.1145/2470654.2481360
54. Nick Taylor, Ursula Hurley, and Philip Connolly. 2016. Making Community. In Proceedings of the 2016 CHI Conference on Human Factors in Computing Systems - CHI '16, 1415-1425. https://doi.org/10.1145/2858036.2858073

55. Carol Thomas. 2004. Disability and Impairment. In Disabling Barriers - Enabling Environments (third edition)., John Swain, Sally French, Colin Barnes and Carol Thomas (eds.). SAGE Publications, 9-16.

56. Carol Thomas. 2012. Theorising disability and chronic illness: Where next for perspectives in medical sociology? Social Theory \& Health 10, 3: 209-228. Retrieved from http:/www.palgravejournals.com/doifinder/10.1057/sth.2012.7

57. Alvin Toffler. 1980. The third wave: The classic study of tomorrow. Bantam, New York.

58. Simo Vehmas and Nick Watson. 2014. Moral wrongs, disadvantages, and disability: a critique of critical disability studies. Disability \& Society 29, 4: 638-650. https://doi.org/10.1080/09687599.2013.831751

59. Jayne Wallace, Peter C Wright, John McCarthy, David Philip Green, James Thomas, and Patrick Olivier. 2013. A design-led inquiry into personhood in dementia. In Proceedings of the SIGCHI Conference on Human Factors in Computing Systems - CHI '13, 2617. https://doi.org/10.1145/2470654.2481363

60. Jacob O. Wobbrock, Shaun K. Kane, Krzysztof Z. Gajos, Susumu Harada, and Jon Froehlich. 2011. Ability-Based Design. ACM Transactions on Accessible Computing 3, 3: 1-27. https://doi.org/10.1145/1952383.1952384

61. Maker Market Study - Maker Faire. Retrieved from http://makerfaire.com/hardware-innovationworkshop/research/ 